\title{
Breakdown of cation vacancies into anion vacancy-antisite complexes on III-V semiconductor surfaces
}

\author{
A. Höglund and S. Mirbt \\ Department of Physics, Uppsala University, Box 530, SE-75121 Uppsala, Sweden \\ C. W. M. Castleton* \\ Department of Physics, Uppsala University, Box 530, SE-75121 Uppsala, Sweden \\ and Department of Materials Chemistry, Uppsala University, Box 538, SE-75121 Uppsala, Sweden \\ M. Göthelid \\ Materialfysik, MAP, ICT, KTH, Electrum 229, SE-16440 Kista, Sweden \\ (Received 27 April 2007; revised manuscript received 5 December 2007; published 22 October 2008)
}

\begin{abstract}
An asymmetric defect complex originating from the cation vacancy on (110) III-V semiconductor surfaces which has significantly lower formation energy than the ideal cation vacancy is presented. The complex is formed by an anion from the top layer moving into the vacancy, leaving an anion antisite-anion vacancy defect complex. By calculating the migration barrier, it is found that any ideal cation vacancies will spontaneously transform to this defect complex at room temperature. For stoichiometric semiconductors the defect formation energy of the complex is close to that of the often-observed anion vacancy, giving thermodynamic equilibrium defect concentrations on the same order. The calculated scanning tunneling microscopy (STM) plot of the defect complex is also shown to be asymmetric in the [1힐 direction, in contrast to the symmetric one of the anion vacancy. This might therefore explain the two distinct asymmetric and symmetric vacancy structures observed experimentally by STM.
\end{abstract}

DOI: $10.1103 /$ PhysRevB.78.155318

PACS number(s): 68.47.Fg, 61.72.J-, 68.37.Ef, 71.15.Mb

\section{INTRODUCTION}

Point defects on the (110) cleavage surfaces of III-V semiconductors have in recent years been studied intensively, using both theoretical and experimental techniques. ${ }^{1-6}$ In contrast to the anion vacancy, there are only a few studies of cation vacancies. In scanning tunneling microscopy (STM) images of the unoccupied states of GaAs (110) surfaces, two features interpreted as being caused by Ga vacancies have been reported: Lengel et $a l^{7}$ observed a dark contrast that appears on an array consisting of about $3 \times 3$ missing unoccupied dangling bonds, whereas Ebert and Urban ${ }^{8}$ and Domke et al. ${ }^{9}$ observed an X-shaped dark contrast consisting of one missing unoccupied dangling bond.

To the knowledge of the authors, there is only one study of In vacancies on InSb, InAs, or InP (110) surfaces. Using dynamic-mode scanning force microscopy, Schwarz et al. ${ }^{10}$ observed In vacancies on $n$-doped InAs (110) surfaces. They observed two As atoms being influenced by a defect in the cation sublattice. They interpreted this defect as an In vacancy but did not rule out the possibility that it might instead be an impurity atom or an antisite defect. The authors are aware of one $a b$ initio study of neutral cation vacancies on GaP (110) surfaces. ${ }^{11}$ In that paper the electronic and structural properties of vacancies on and below the surface were studied. It was found that the surface and subsurface vacancies maintain the $C_{1 h}$ symmetry of the defect-free (110) surface, i.e., there remains a single mirror plane along the [1 10$]$ direction. In bulk GaAs, earlier calculations have shown that the cation vacancy is metastable with respect to the formation of a defect complex consisting of an anion vacancy and an anion antisite. ${ }^{12-14}$
We here present $a b$ initio calculations on cation vacancies on the InSb, InAs, InP, GaP, and GaAs (110) surfaces in which we find the ideal isolated cation vacancy to be unstable against the formation of an asymmetric defect complex. This complex is formed by an anion from the top layer moving in to occupy the cation vacancy site, giving rise to an anion antisite-anion vacancy defect complex, which breaks the $C_{1 h}$ symmetry. The STM signature of this defect complex consists of an "anion vacancy" in the occupied state image and a "cation vacancy" in the unoccupied state image. Moreover, the concentrations of the often-observed pure anion vacancies and of this different defect complex are calculated to be similar under stoichiometric conditions. Therefore the interpretation of STM images of both anion and cation vacancies on III-V semiconductors may need to be revised.

\section{COMPUTATIONAL METHOD}

We use plane-wave $a b$ initio density-functional theory ${ }^{15}$ (DFT) within the local-density approximation (LDA) together with ultrasoft pseudopotentials ${ }^{16,17}$ using the VASP code. ${ }^{18}$ Exchange and correlation potentials are described by the functional of Ceperley and Alder as parametrized by Perdew and Zunger. ${ }^{19}$ The $4 d$ electrons of the indium and gallium atoms are treated as core electrons. For charged defects, a uniform compensating background is incorporated to maintain the charge neutrality of the supercell. ${ }^{20}$

The surface calculations were performed using a sevenlayer-thick $2 \times 4$ periodic slab which is pseudo-hydrogenterminated on one side. Increasing the slabs to $4 \times 4$ surface cells has been shown to change the formation energies by 


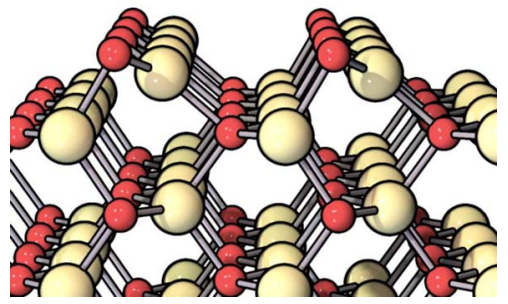

(a)Clean surface

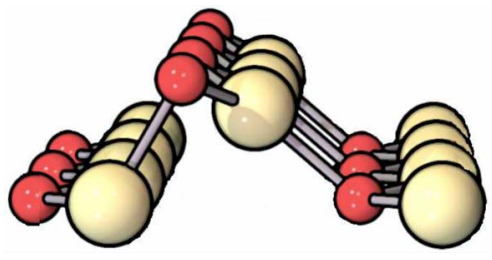

(b)Clean surface - Close-up

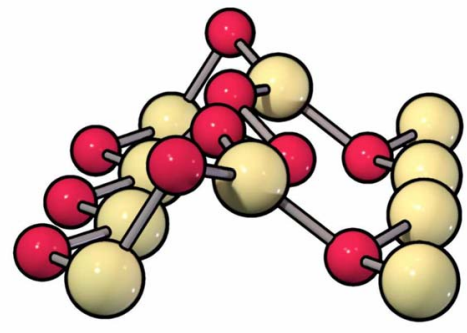

(c)Cation vacancy, $\mathrm{V}_{\mathrm{C}}$

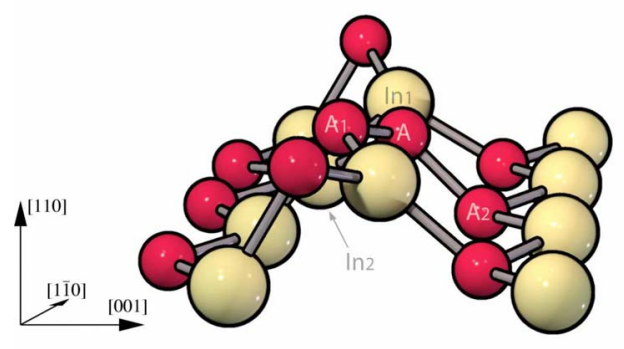

(d)Defect complex, $\mathrm{V}_{\mathrm{A}}-\mathrm{A}_{\mathrm{C}}$

FIG. 1. (Color online) Structures of (a) the reconfigured clean (110) surface, (b) a closeup of the ideal surface, (c) the ideal cation vacancy, and (d) the defect complex. The structures shown are InP but are representative of all presented III-V semiconductors. Cations are represented by light (beige) spheres and anions by dark (red) spheres.

less than $0.04 \mathrm{eV}^{6}$ A $2 \times 2 \times 2$ Monkhorst-Pack selection was used for the $k$-point sampling.

During the relaxations the top four layers are allowed to relax fully while layers five to seven are fixed at their bulk positions. The migration barriers were calculated using the nudged-elastic-band method (NEB). ${ }^{21}$ The optimized LDA values were used for the lattice constants and were calculated to be 5.827, 6.013, 6.430, 5.390, and 5.601 $\AA$ for InP, InAs, $\mathrm{InSb}, \mathrm{GaP}$, and $\mathrm{GaAs}$, respectively.

\section{RESULTS}

We have previously compared the defect formation energies of the native defects between bulk and surface. ${ }^{6}$ In that comparison we included the defect formation energy of the cation vacancy at the surface. The structure of that cation vacancy, however, turns out to be more complex than expected. Surprisingly, the simple cation vacancy, defined as the absence of only one surface cation, is not stable at the surface. Instead the removal of one cation gives rise to the formation of a defect complex: an anion vacancy bound to an anion antisite $\left[V_{A}-A_{C}\right]{ }^{22}$

\section{A. Defect complex structure}

In Figs. 1(a) and 1(b) we show two different views of the structure of the clean surface, plus (c) the ideal cation vacancy and (d) the $\left[V_{A}-A_{C}\right]$ in InP. In the complex in Fig. 1(d), the void left by the missing cation is occupied by a surface anion, which means that an anion antisite and an anion surface vacancy are formed. This creates two anion dimers and one cation dimer independent of the charge state of $\left[V_{A}-A_{C}\right]$. One anion dimer lies within the surface and the other anion dimer and the cation dimer are directed toward the subsurface layer. For the -1 charge state the extra electron is used to create a bond between the antisite and the subsurface cation closest to the antisite (In2) (see Fig. 1), but for the 0 and +1 charge states this bond is absent.

The anion antisite $A$ is located about $0.5 \AA$ deeper into the surface than its anion nearest neighbor $A 1$ (see $\Delta z_{A 1-A}$ in Table I). Compared to its cation nearest neighbor, the anion antisite is located about $0.1 \AA$ deeper into the surface. The cation in the subsurface layer $(C 2)$ relaxes by about $0.25 \AA$ out of the subsurface layer along the $z$ direction (see $\Delta z_{C 2}$ in Table I) and by about the same amount toward the antisite along the $[1 \overline{1} 0]$ direction. In the $[001]$ direction $C 2$ relaxes by about $0.8 \AA$.

\section{B. Stability}

In Fig. 2 we show the calculated defect formation energies for the ideal cation vacancy and the defect complex in InSb, InAs, InP, GaP, and GaAs. (The formation energies are plotted over the experimental band gap. For further discussion see Ref. 6.) For InSb, InAs, and InP the formation energies of all other native defects, as reported earlier, ${ }^{6}$ are also shown in the background as a reference. $\left[V_{A}-A_{C}\right]$ and $V_{C}$ have an identical dependence on the chemical potential. The formation energy of $V_{C}$, corresponding purely to the removal of a cation, is seen to be much higher than the formation energy of the defect complex, $\left[V_{A}-A_{C}\right]$. This can be understood by considering the two components of the complex (the anion vacancy and the anion antisite) separately and comparing them to the ideal cation vacancy. 
TABLE I. Bond length $d$ and the change in distance due to the relaxations of the nearest-neighbor atoms of the anion antisite $A$ (in $\AA$ ). $A X$ refers to the anion and $C X$ to the cation at the surface $(X=1)$ or in the subsurface $(X=2) . \Delta z_{A 1-A}$ is the difference in height between the anion antisite and its nearest-neighbor anion.

\begin{tabular}{|c|c|c|c|c|c|c|}
\hline & $q$ & $d_{A 1-A}$ & $d_{A 2-A}$ & $d_{C 1-C 2}$ & $d_{A-C 2}$ & $\Delta z_{A 1-A}$ \\
\hline \multirow[t]{3}{*}{ InP } & +1 & 2.12 & 2.16 & 2.92 & 3.60 & 0.40 \\
\hline & 0 & 2.15 & 2.18 & 2.87 & 3.26 & 0.51 \\
\hline & -1 & 2.19 & 2.21 & 2.85 & 2.81 & 0.53 \\
\hline \multirow[t]{2}{*}{ InAs } & 0 & 2.42 & 2.43 & 2.95 & 3.00 & 0.50 \\
\hline & -1 & 2.44 & 2.45 & 2.88 & 2.81 & 0.53 \\
\hline $\mathrm{InSb}$ & -1 & 2.84 & 2.81 & 2.90 & 2.95 & 0.54 \\
\hline \multirow[t]{3}{*}{$\mathrm{GaP}$} & +1 & 2.16 & 2.16 & 2.68 & 3.41 & 0.28 \\
\hline & 0 & 2.18 & 2.18 & 2.59 & 3.12 & 0.38 \\
\hline & -1 & 2.21 & 2.20 & 2.55 & 2.61 & 0.47 \\
\hline \multirow[t]{2}{*}{ GaAs } & 0 & 2.45 & 2.43 & 2.66 & 2.79 & 0.44 \\
\hline & -1 & 2.47 & 2.44 & 2.58 & 2.62 & 0.48 \\
\hline
\end{tabular}

First, we discuss the low formation energy of antisites. For an antisite, the nearest-neighbor distances can be relaxed to the equilibrium value without affecting the remaining crystal at all. Therefore the antisite could, in principle, be as low in energy as the ideal surface. As expected, the formation energies of the antisites are also very low, as seen in Fig. 2. The largest contribution to the formation energy of the complex therefore comes from the anion vacancy.

Second, we compare the formation energy of a surface anion vacancy with that of the unstable cation surface vacancy, i.e., $V_{C}$. A vacancy gives rise to the formation of dimers: an anion vacancy leads to the formation of cation dimers and a cation vacancy leads to the formation of anion dimers. For example, for the -1 charged anion vacancy and the +1 charged cation vacancy, four electrons occupy two vacancy states. The difference in formation energy is then not determined by a difference in the number of electrons but by the difference in electron screening. The anion valence electrons are less screened by the core electrons than the cation valence electrons. This implies that more electrons can contribute to an anion dimer bond than to a cation dimer bond, or in other words the overlap between two anions is larger than between two cations. Therefore the difference in formation energy between $V_{C}$ and $V_{A}$ increases with the ionicity of the III-V compound. Starting with the material with the largest ionicity, the difference in formation energy between $V_{C}$ and $\left[V_{A}-A_{C}\right]$ for the neutral charge state is equal to $0.85,0.71,0.50,0.15$, and $0.10 \mathrm{eV}$ for InP, GaP, InAs, GaAs, and $\mathrm{InSb}$, respectively (see Fig. 2).

\section{Migration barrier}

In order to consider dynamic effects in the system, we start by calculating the migration barrier between the ideal cation vacancy, $V_{\mathrm{In}}$, and the defect complex, $\left[V_{A}-A_{C}\right]$. NEB was used to find the minimum-energy path and linear interpolations of the coordinates along the migration path were used as the initial guesses. Due to the computational time costs, these calculations were only performed for the mate- rials with the largest and smallest differences in ionicity, as measured by the difference in the covalent radii, i.e., InP and GaAs. From physical arguments the other three materials are expected to have intermediate values for the migration barrier.

The energy along the migration path and the barriers are shown in Fig. 3. In InP a migration barrier of $0.41 \mathrm{eV}$ is found and in GaAs an almost negligible barrier of $0.05 \mathrm{eV}$ is found. Starting from transition state theory, assuming a typical prefactor of $10^{12} \mathrm{~s}^{-1}$ (taken from Ref. 23) and an onset for the reaction coordinate of $k=1 \mathrm{~s}^{-1}$, the onset temperature for a transition from $V_{C}$ to $\left[V_{A}-A_{C}\right]$ is $160 \mathrm{~K}$ in $\mathrm{InP}$ and 20 $\mathrm{K}$ in GaAs. ${ }^{24,25}$ In other words, at room temperature any measurable concentration of ideal cation vacancies will have transformed to the more stable complex structure. We therefore conclude that $V_{\text {In }}$ does not occur on III-V semiconductor surfaces at normal temperatures other than in nonequilibrium systems. This conclusion is based on the higher formation energy of $V_{C}$ and on the instability of $V_{C}$ to the formation of $\left[V_{A}-A_{C}\right]$. We generalize our statement, expecting it to be valid for all III-V semiconductors, because the underlying physics is common to all III-V semiconductor surfaces.

We note that the cation vacancy in bulk GaAs has earlier been calculated to be metastable. ${ }^{12-14}$ In agreement with the calculations for bulk GaAs, we also report here that for bulk InP the $\left[V_{\mathrm{P}}-P_{\text {In }}\right]$ complex is more stable than $V_{\text {In }}$ for $p$ type up to semi-insulating conditions. In a computational study, Schwarz et al. ${ }^{11}$ found the Ga vacancy on GaP (110) to maintain the $C_{1 h}$ symmetry. This can be shown to be in agreement with what is found here, as follows. It can be seen from Fig. 1 (c) that the mirror symmetry in the $[1 \overline{1} 0]$ direction is maintained. Generalizing the ideal cation vacancy structures found in this study, for all five materials and all charge states, the void of the cation vacancy is filled up by a symmetric inward relaxation of all three nearest-neighbor anions and in some cases the inward movement of the anion in the second layer is also more pronounced (which still preserves the $C_{1 h}$ symmetry). The defect complex, on the other hand, is created when the void of the cation vacancy is instead filled by an 

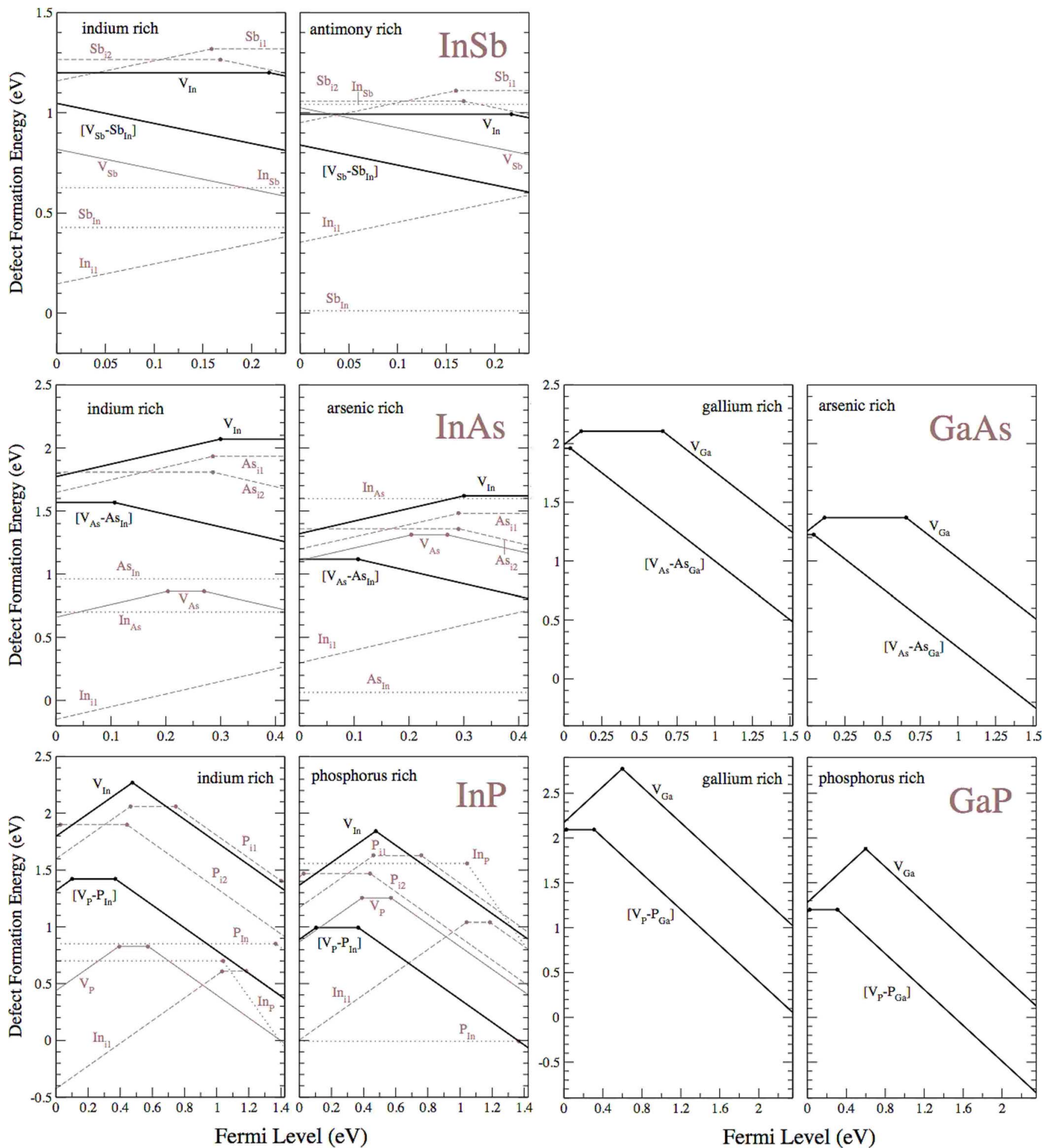

FIG. 2. (Color online) Formation energies of the cation vacancy $V_{C}$ and of the defect complex $\left[V_{A}-A_{C}\right]$ on the (110) surface of InSb, InAs, InP, GaP, and GaAs as functions of the Fermi level under cation-rich conditions (left panel of each figure) and under anion-rich conditions (right panel of each figure). Gray lines correspond to the defect formation energies of the other native point defects. Data taken from Ref. 6.

anion from the top layer. Therefore the $C_{1 h}$ symmetry is broken as seen in Fig. 1(d). We have now shown that the complex is lower in energy. But for GaP there will be a nonzero migration barrier, which is most likely why Schwarz et al. ${ }^{11}$ did not find this structure.

\section{Simulated STM}

Next, we discuss the calculated STM image of $\left[V_{A}-A_{C}\right]$. In Fig. 4 we show the occupied and unoccupied calculated STM images for bias voltages of -2 and $+2 \mathrm{eV}$, respec- 


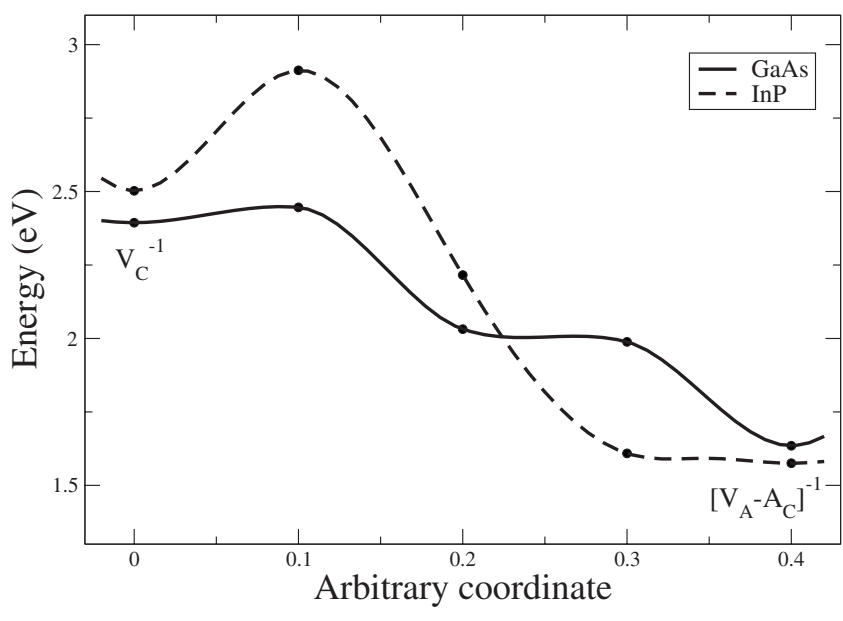

FIG. 3. Calculated energy barrier when going from the cation vacancy to the anion vacancy-anion antisite defect complex.

tively. In the unoccupied state image the defect complex $\left[V_{A}-A_{C}\right]$ gives rise to a single missing spot in the cation sublattice. In the occupied state image it gives rise to a missing spot in the anion sublattice. From experimental STM images it is thus very hard to distinguish between the existence of $\left[V_{A}-A_{C}\right]$ and that of $V_{A}$. To distinguish between the two, the same area needs to be viewed at both negative and positive biases, and the two images need to be aligned very precisely for comparison. This implies that the interpretation of many experimentally observed anion vacancies on III-V semiconductors may need to be revised.

For all stable charge states, the anion antisite and $A 1(A 1$ refers to the anion in the surface layer which is nearest neighbor with the anion antisite) each has an occupied dangling bond. The dangling bond on $A 1$ appears weaker in the STM picture (see Fig. 4) and the dangling bond on the antisite is not seen at all, independent of the charge state of $\left[V_{A}-A_{C}\right]$. The reason is that the height of the dangling bond relative to the rest of the surface will affect the STM image. The dangling bond on $A 1$ is located about $0.2 \AA$ lower than the other anion dangling bonds. This explains the asymmetry in Fig. 4 between the occupied STM spot at $A 1$ and the next closest occupied spot. The dangling bond on the anion antisite is about $0.5 \AA$ lower than the other anion dangling bonds, which explains why it is not seen. For the 0 charge state, the anion antisite has a partly occupied dangling bond directed toward the subsurface. For the +1 charge state the anion antisite has one empty dangling bond directed toward the subsurface. The calculated STM images for the 0 and +1 charge states (not shown here) have the same signature as the STM image of the -1 charge state because the charge variation in the occupation of one of the antisite dangling bonds takes place low in the surface and is therefore not seen in the STM picture.

In the following we compare experimental STM images of anion vacancies with the STM signature of the defect complex presented here. We start with the $\operatorname{In} X$ materials. For $n$-type InSb, InAs, and InP the configuration of the experimentally observed anion vacancy is nonsymmetric with a -1 charge state $e^{26-28}$ but the calculated $V_{A}$ is symmetric with a -1 charge state. ${ }^{5}$ In contrast to the anion vacancy, the configu- ration of the complex is found to be always nonsymmetric and for $n$-type conditions it is calculated to have a -1 charge state. From anion-rich conditions up to about stoichiometric conditions, the concentration of $\left[V_{A}-A_{C}\right]$ should be higher than that of $V_{A}$ except in strongly $p$-type material in InP and InAs (see Fig. 2). We therefore conclude that for $n$-type III-V semiconductor surfaces under anion-rich conditions (up to about stoichiometric conditions), the experimentally observed anion vacancies are more likely to correspond to $\left[V_{A}-A_{C}\right]$ than to $V_{A}$. This is confirmed by comparing the occupied state STM image of a negatively charged missing spot in the anion sublattice on an $n$-type InP (110) surface with our calculated image of $\left[V_{A}-A_{C}\right]$. In Fig. 2(e) of Ref. 26, Ebert et al. showed a STM image that had exactly the same signatures as our calculated STM image. The asymmetry between the two anion spots closest to the missing spots is clearly visible.

For $p$-type InP the configuration of the experimentally observed anion vacancy is symmetric, with $\mathrm{a}+1$ charge state $26,29,30$ but the configuration of the calculated $V_{A}$ is nonsymmetric with a +1 charge state for both InP and InAs. ${ }^{5}$ The $\left[V_{A}-A_{C}\right]$ is calculated to have a +1 charge state for InP, a 0 charge state for InAs, and a -1 charge state for InSb (see Fig. 2 ). To the authors' knowledge there are no experimental reports studying surface vacancies on $p$-type InAs and InSb. For $p$-type conditions and from cation-rich conditions up to about stoichiometry, the concentration of $V_{A}$ is calculated to be larger than that of $\left[V_{A}-A_{C}\right]$ for InSb, InP, and InAs. The disagreement between experiment and theory in this limit has two possible explanations. The first explanation is that thermal flipping between two degenerate distorted $V_{A}$ structures averages out over STM measurement time scales to leave an apparently symmetric image. Simulated STM images based on this idea by Ebert et al. ${ }^{30}$ do indeed resemble the experimental images. However, an even better resemblance is obtained in simulated STM images using the second explanation, namely, hydrogen adsorption on a surface anion. ${ }^{31}$ Which of the two explanations is correct, or indeed, if both are correct under different experimental conditions, is so far unclear.

For $p$-type conditions, approaching the anion-rich limit, the concentration of $\left[V_{A}-A_{C}\right]$ should be higher than that of $V_{A}$ for InSb. For InAs and InP the concentration of $V_{A}$ is calculated to be about the same as for the complex.

Whitman et al. ${ }^{28}$ conducted a STM study on an $n$-type InSb (110) surface, which confirms the results we present here. They observed two types of Sb vacancies: one where there is a missing spot in the occupied STM image, but no perturbation is observed in the unoccupied STM image. They interpreted this as a simple anion vacancy. In the other there is again a missing spot in the occupied STM image, but in addition the adjacent cation is missing in the unoccupied STM image. According to our calculations we reinterpret their results in the following way: At stoichiometric conditions the concentration of $V_{A}$ and $\left[V_{A}-A_{C}\right]$ should be similar on InSb. The first observed vacancy type corresponds to a simple anion vacancy, in agreement with their interpretation. The second observed vacancy type we instead interpret as $\left[V_{A}-A_{C}\right]$.

We turn now to $\mathrm{Ga} X$. We are not aware of any relevant experimental studies on GaP but, as mentioned in Sec. I, 


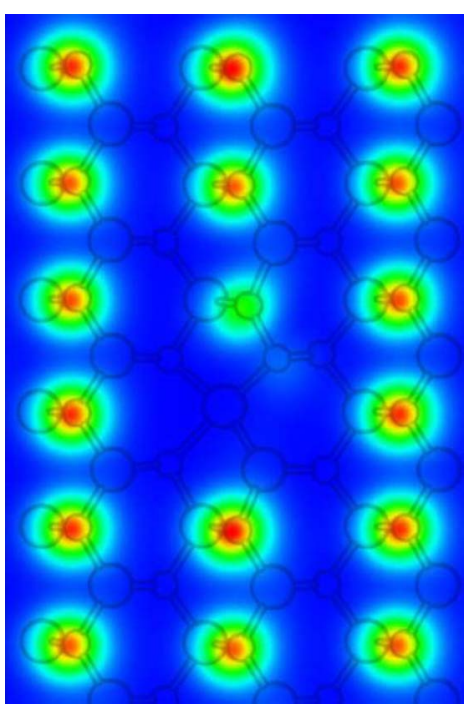

(a) $\left[\mathrm{V}_{\mathrm{P}}-\mathrm{P}_{\mathrm{In}}\right]^{-1}$

$(-2.0 \mathrm{eV})$

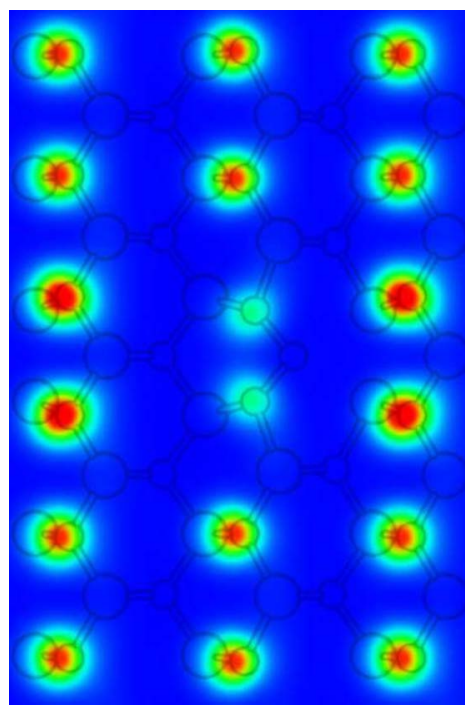

(c) $\mathrm{V}_{\mathrm{In}}^{-1}(-2.0 \mathrm{eV})$

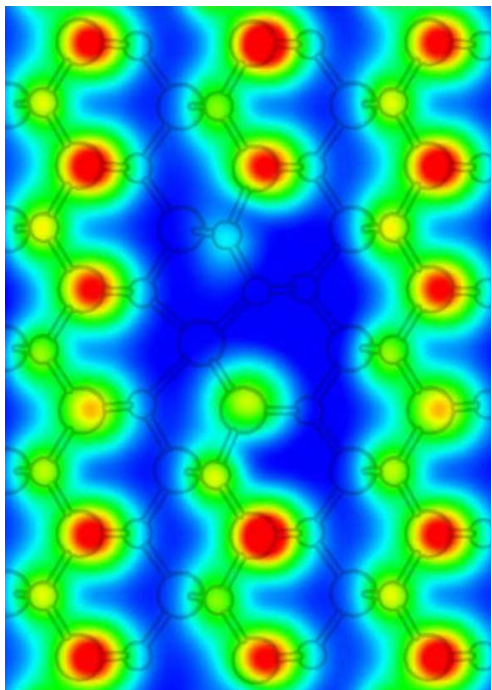

(b) $\left[\mathrm{V}_{\mathrm{P}}-\mathrm{P}_{\text {In }}\right]^{-1}$

$(+2.0 \mathrm{eV})$

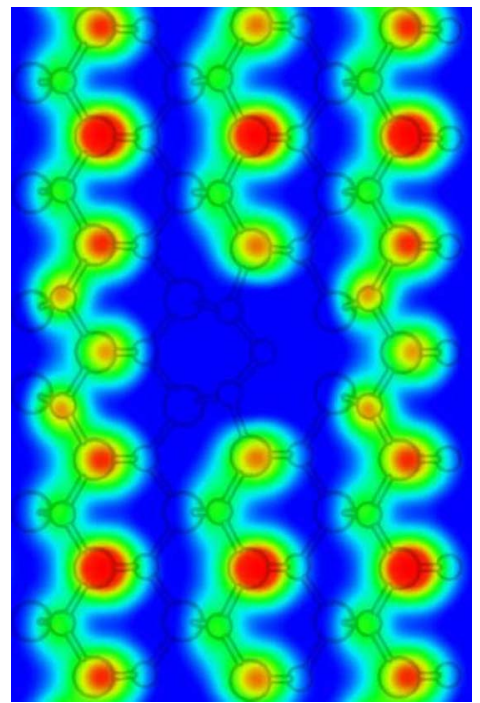

(d) $\mathrm{V}_{\text {In }}^{-1}(+2.0 \mathrm{eV})$
FIG. 4. (Color online) Calculated STM image of $\left[V_{A}-A_{C}\right]$ (top) and $V_{C}$ (bottom) on $\operatorname{InP}(110)$, both for charge state -1 . The images to the left (right) show occupied (empty) states corresponding to a bias voltage of $-2.0 \mathrm{eV}(+2.0 \mathrm{eV})$. Atomic structures are shown in the background. studies have been made on GaAs. Here, a dark contrast on an array of about $3 \times 3$ missing unoccupied dangling bonds has been interpreted as the signature of a Ga vacancy. ${ }^{7}$ That signature does not agree with the signature of the complex, but we suggest that signature to be caused by hydrogen adsorption. ${ }^{31}$ Another group interpreted the X-shaped dark contrast to be the signature of a Ga vacancy. ${ }^{30}$ Neither experimental image agrees with the signature of the complex, but another group has earlier interpreted the $\mathrm{X}$ signature to be caused by a $\left[\mathrm{V}_{\mathrm{Ga}}-\mathrm{Si}_{\mathrm{Ga}}\right]$ defect complex. ${ }^{9}$ Further studies may now be needed to clarify the origin of the X-shaped signature.

\section{CONCLUSIONS}

In summary, we have shown that the removal of a single cation on III-V semiconductor surfaces is unstable against the formation of a defect complex consisting of an anion vacancy and an anion antisite. This defect complex gives rise to a STM picture which may appear like an anion vacancy. We suggest that many of the anion vacancies observed by STM are caused by this defect complex. This can, in many cases, resolve the disagreement between theory and experiment regarding the symmetry of the anion vacancy. 
*Present address: School of Science and Technology, Clifton Campus, Nottingham Trent University, Nottingham, NG11 8NS, UK.

${ }^{1}$ G. P. Srivastava, Rep. Prog. Phys. 60, 561 (1997).

${ }^{2} \mathrm{Ph}$. Ebert, Surf. Sci. Rep. 33, 121 (1999).

${ }^{3} \mathrm{Ph}$. Ebert, Curr. Opin. Solid State Mater. Sci. 5, 211 (2001).

${ }^{4}$ M. C. Qian, M. Göthelid, B. Johansson, and S. Mirbt, Phys. Rev. B 67, 035308 (2003).

${ }^{5}$ M. C. Qian, M. Göthelid, B. Johansson, and S. Mirbt, Phys. Rev. B 66, 155326 (2002).

${ }^{6}$ A. Höglund, C. W. M. Castleton, M. Göthelid, B. Johansson, and S. Mirbt, Phys. Rev. B 74, 075332 (2006).

${ }^{7}$ G. Lengel, R. Wilkins, G. Brown, and M. Weimer, J. Vac. Sci. Technol. B 11, 1472 (1993).

${ }^{8} \mathrm{Ph}$. Ebert and K. Urban, Phys. Rev. B 58, 1401 (1998).

${ }^{9}$ C. Domke, Ph. Ebert, M. Heinrich, and K. Urban, Phys. Rev. B 54, 10288 (1996).

${ }^{10}$ A. Schwarz, W. Allers, U. D. Schwarz, and R. Wiesendanger, Phys. Rev. B 61, 2837 (2000).

${ }^{11}$ G. Schwarz, A. Kley, J. Neugebauer, and M. Scheffler, Phys. Rev. B 58, 1392 (1998).

${ }^{12}$ G. A. Baraff and M. Schlüter, Phys. Rev. Lett. 55, 2340 (1985).

${ }^{13}$ Y. Bar-Yam and J. D. Joannopoulos, Phys. Rev. Lett. 56, 1213 (1986).

${ }^{14}$ M. Bockstedte and M. Scheffler, Z. Phys. Chem. 200, 195 (1997).

${ }^{15}$ W. Kohn and L. J. Sham, Phys. Rev. 140, A1133 (1965).

${ }^{16}$ D. Vanderbilt, Phys. Rev. B 41, 7892 (1990).

${ }^{17}$ G. Kresse and J. Hafner, J. Phys.: Condens. Matter 6, 8245
(1994).

${ }^{18}$ G. Kresse and J. Furthmüller, Comput. Mater. Sci. 6, 15 (1996).

${ }^{19}$ J. P. Perdew and A. Zunger, Phys. Rev. B 23, 5048 (1981).

${ }^{20}$ M. Leslie and M. J. Gillian, J. Phys. C 18, 973 (1985).

${ }^{21}$ G. Mills, H. Jonssón, and G. K. Schenter, Surf. Sci. 324, 305 (1995).

${ }^{22}$ Note that in Ref. 6 the defect complex was labeled as $V_{\text {In }}$.

${ }^{23}$ L. Xu, G. Henkelman, C. T. Campbell, and H. Jónsson, Surf. Sci. 600, 1351 (2006).

${ }^{24}$ H. Jónsson, Annu. Rev. Phys. Chem. 51, 623 (2000).

${ }^{25}$ J. Song, L. R. Corrales, G. Kresse, and H. Jonssón, Phys. Rev. B 64, 134102 (2001).

${ }^{26} \mathrm{Ph}$. Ebert, K. Urban, and M. G. Lagally, Phys. Rev. Lett. 72, 840 (1994).

${ }^{27}$ A. Depuydt, N. S. Maslova, V. I. Panov, V. V. Rakov, S. V. Savinov, and C. Van Haesendonck, Appl. Phys. A: Mater. Sci. Process. 66, S171 (1998).

${ }^{28}$ L. J. Whitman, J. A. Stroscio, R. A. Dragoset, and R. J. Celotta, Phys. Rev. B 42, 7288 (1990); J. Vac. Sci. Technol. B 9, 770 (1991).

${ }^{29}$ M. Heinrich, Ph. Ebert, M. Simon, K. Urban, and M. G. Lagally, J. Vac. Sci. Technol. A 13, 1714 (1995).

${ }^{30} \mathrm{Ph}$. Ebert, K. Urban, L. Aballe, C. H. Chen, K. Horn, G. Schwarz, J. Neugebauer, and M. Scheffler, Phys. Rev. Lett. 84, 5816 (2000).

${ }^{31}$ C. W. M. Castleton, A. Höglund, M. Göthelid, M. Qian, and S. Mirbt (unpublished). 